\title{
COMBINATION OF GEO-MAGNETIC JERKS WITH UPDATED ESMGFZ EFFECTIVE ANGULAR MOMENTUM FUNCTIONS FOR THE MODELLING OF POLAR MOTION EXCITATION
}

\author{
Cyril RON $^{1)}$ *, Jan VONDRÁK ${ }^{1)}$, Robert DILL ${ }^{2)}$ and Yavor CHAPANOV ${ }^{3)}$ \\ 1) Astronomical Institute, Czech Academy of Sciences; Bočni II 1401, Prague, Czech Republic \\ ${ }^{2)}$ Helmholtz Centre - GeoForschungsZentrum; Telegrafenberg, Potsdam, Germany \\ ${ }^{3)}$ Climate, Atmosphere and Water Research Institute, Bulgarian Academy of Sciences; Sofia, Bulgaria
}

*Corresponding author's e-mail: ron@asu.cas.cz

\begin{tabular}{l} 
ARTICLE INFO \\
\hline Article history: \\
Received 13 May 2019 \\
Accepted 27 August 2019 \\
Available online 9 October 2019
\end{tabular}

Keywords:

Polar motion

Geophysical excitation

Geomagnetic jerks

\begin{abstract}
We use numerical integration of Brzeziński's broad-band Liouville equations with the new atmospheric/oceanic excitations provided by GFZ Potsdam to calculate their effect on polar motion. There exist several studies hinting that geomagnetic jerks, sudden changes of intensity of geomagnetic field, can also affect polar motion. We concentrate our efforts to study possible influence of geomagnetic jerks on temporal changes of polar motion. We demonstrate that the fit between integrated and polar motion observed by space geodesy improves substantially, if the influence of geomagnetic jerks is added to excitations by geophysical fluids. We also applied additional excitations due to the continental hydrosphere and barystatic sea-level variations. Although these effects complete the geophysical fluids system, their adding could not improve the agreement between integrated and observed polar motion.
\end{abstract}

\section{INTRODUCTION}

It was demonstrated earlier by many authors (e.g. Barnes et al., 1983; Brzezinski, 1994; Gross, $2005,2009)$ that excitations by geophysical fluids (atmosphere, oceans) play dominant role in polar motion and rotational velocity of the Earth. Much smaller effect can be observed also in nutation (Malkin, 2013; Vondrák and Ron, 2017, 2019). In our previous paper (Vondrák et al., 2017), we used excitation series derived by GFZ on base of atmospheric data ERA40 and ERAInterim of the European Centre for Medium-Range Weather Forecasts (ECMWF) and oceanic data based on the Ocean Model for Circulation and Tides (OMCT) (Dobslaw et al., 2010) both given at 6-h intervals. The influence of excitation of the continental hydrosphere has not been used yet in our solution although the hydrospheric effective angular momentum functions (EAMF) were available. We supplemented the ERA/OMCT series with the geomagnetic excitation due to geomagnetic jerks (GMJ) and we determined the new period $432.86 \pm 0.04$ days and quality factor of Chandler wobble $35 \pm 0.3$ considering these geophysical excitations.

New series of geophysical fluids EAMF has been provided by the Earth System Model group of GeoForschungsZentrum, Potsdam (ESM GFZ) at the IERS server in 2018 (Dobslaw and Dill, 2018), that contain the atmospheric and oceanic EAMF at 3-h intervals. The oceanic EAMF are derived newly from the Max Planck Institute Ocean model (MPIOM) and the EAMF of atmosphere, ocean and hydrosphere have been supplemented with the barystatic sea level EAMF that balances the global water budget of atmosphere and terrestrial water storage as additional contribution in the ocean. Thus we decided to make the new integration using the ESM GFZ data.

\section{METHOD}

Polar motion excited by geophysical fluids can be derived from numerical integration of Brzeziński's broad-band Liouville equation (Brzeziński, 1994) for a two-layer Earth model, that are expressing the reaction of polar motion $p$ to excitations $\chi$. It is written (in complex form) as follows:

$$
\begin{aligned}
\ddot{p}- & i\left(\sigma_{c}+\sigma_{f}\right) \dot{p}-\sigma_{c} \sigma_{f} p= \\
& =-\sigma_{c}\left\{\sigma_{f}\left(\chi_{p}+\chi_{w}\right)+\sigma_{c}\left(a_{p} \chi_{p}+a_{p} \chi_{w}\right)+\right. \\
& \left.+i\left[\left(1+a_{p}\right) \dot{\chi}_{p}+\left(1+a_{w}\right) \dot{\chi}_{w}\right]\right\}
\end{aligned}
$$

where $p=x-\mathrm{i} y$ is the polar motion, $\sigma_{c}, \sigma_{f}$ are the two main resonance complex frequencies - prograde Chandler and retrograde free core nutation (RFCN) expressed in terrestrial frame, $\chi_{p}, \chi_{w}$ are the EAMF of pressure and wind terms, $a_{p}=0.0920, a_{w}=2.628 \times 10^{-4}$ are numerical constants expressing different reaction of the Earth on pressure and wind terms taken from Koot and de Viron (2011). The value of RFCN frequency has been fixed by adopting the value used 
to derive the most recent model of nutation (Mathews et al., 2002), $\sigma_{f}=-6.31498+0.000153 \mathrm{iad} /$ day. The Chandler frequency was searched using the equation $\sigma_{c}=\Omega(1+i / 2 Q) / T$ where $\Omega=6.30038 \mathrm{rad} /$ day is the mean speed of Earth's rotation, $T$ is the period of Chandler wobble, and $Q$ is its quality factor.

Within last decade it was noticed that sudden jumps of phase or amplitude of Earth orientation are somehow correlated with GMJ. Since the mechanism of transferring the changes of geomagnetic field to Earth's orientation parameters remains unknown, we are unable to compute the excitation of GMJ directly from known changes of geomagnetic field. Instead, we model it by impulse-like function (Eq. 2), centered at GMJ epochs, whose amplitude is estimated to yield the best fit between the integrated and observed polar motion

$$
\chi_{G M J}=\frac{a}{2}\left(1+\cos \frac{2 \pi\left(t-t_{0}\right)}{\Delta}\right),
$$

where $a$ is the estimated complex amplitude, $t_{0}$ is the epoch of the GMJ, $\Delta$ the width of the interval of acting geomagnetic excitation $\Delta=200$ days which is applied in the interval $t_{0} \pm \Delta / 2$. We found this as optimal value already in our preceding study (Vondrák and Ron, 2015). In that paper we also tested a possible shift of the GMJ epochs either before or after the epochs listed in literature and the best agreement has been found for the exact values of epochs. See Vondrák and Ron $(2015,2016)$ for more details.

For integrating Eq. (1) we need to choose four integration constants, the initial position of pole $p_{0}$ and its first time derivative $\dot{p}_{0}$. The solution contains two damped free motions: prograde Chandler motion with 14-month period, and retrograde quasi-diurnal RFCN which is by definition forbidden in polar motion. Consequently, we impose a constraint of initial value of the first derivative to suppress this motion $\dot{p}_{0}=i\left(\sigma_{c}-\sigma_{f}\right) p_{0}$, and choose only $p_{0}$. To integrate the equations we use standard fourth-order Runge-Kutta procedure rk4 as given by Press et al. (1992) with 3-hour steps modified into complex form.

We have used a faster procedure based on successive approximations. We do not integrate Eq. (1) for many combinations of the period $T$, and quality factor $Q$ as in Vondrák et al. (2017). Instead, we choose some initial values of Chandler wobble parameters $T, Q$, find the best-fitting initial pole position $p_{0}$ and amplitudes $a$ of GMJ excitation in second variant and during the process of integration we also calculate numerically partial derivatives of the pole position with respect to estimated unknown Chandler wobble parameters. They are then used to form observation equations and normal equations whose solution yields the improved values of the unknowns and their formal uncertainties. If the unknowns differ from the initial ones significantly, we use these as new initial values and repeat whole procedure until convergence is achieved.

\section{DATA USED}

We use the following data, covering the interval 1980.0-2018.5. The ESM GFZ data are available even for earlier period, but the wind term before 1980 has twice higher amplitudes than after 1980 and it could bring an inhomogeneity in our solution. This difference comes from the change of method of meteorological data collection in the ECMWF which is the source for new ESM GFZ atmospheric EAMF series. Very detailed description and figures of all used EAMFs is available in the Product Description Document on the web pages of ESM GFZ (Dobslaw and Dill, 2019).

The atmospheric and oceanic excitations have been taken in the form of EAMF $\chi_{p, w}$ that are available at 3-hour resolution from the ESMGFZ server (Dobslaw and Dill, 2018). The oceanic data are based on the model MPIOM (Jungclaus et al., 2013) rather than former ocean model OMCT (Thomas, 2002) and the comparison between integration and observed polar motion shows better agreement with newer model. The hydrological EAMF is calculated from terrestrial water storage as given by the Land Surface Discharge Model (LSDM; Dill, 2008) in daily intervals. The barystatic sea-level EAMF are calculated from sea-level changes balancing the excess water mass of atmosphere and continental hydrosphere to ensure global mass conservation among all geophysical fluids. The barystatic sea-level also considers small effects coming from loading and self-attraction by solving explicitly the sea-level equation (Tamisiea, 2010). These excitations were not used in our previous solutions. The EMS GFZ solution represents now globally mass consistent set of EAMFs and we decided to apply all available excitation functions in addition to only use atmospheric and oceanic EAMF. For the integration we smoothed the data to keep the periods longer than one day and interpolated them into 3-hour intervals. The new barystatic excitations series appeared in the ESM GFZ server in March 2019 (Dobslaw and Dill, 2019).

For observed polar motion we used C04 solution, provided by the International Earth Rotation and Reference Systems Service (IERS) in daily intervals. These series were filtered (Vondrák, 1977) to remove the components with periods longer than 6000 days that are known to be caused by other effects than geophysical fluids.

The epochs of 9 irregularly spaced GMJ events have been used as they are reported by different authors: the epochs and sources being 1986.0, 1991.0, 1999.0 (Mandea et al., 2010), 1994.0 (Malkin, 2013), 2003.5, 2004.7 (Olsen and Mandea, 2008), 2007.5, 2011.0 (Chuillat and Maus, 2014), and 2014.0 (Brown et al., 2016).

\section{RESULTS, CONCLUSIONS}

The integrations were done in several combinations of excitations atmospheric and oceanic (AO), hydrospheric $(\mathrm{H})$, barystatic sea-level (S) and geomagnetic jerks $(\mathrm{G})$. The solutions were done with 

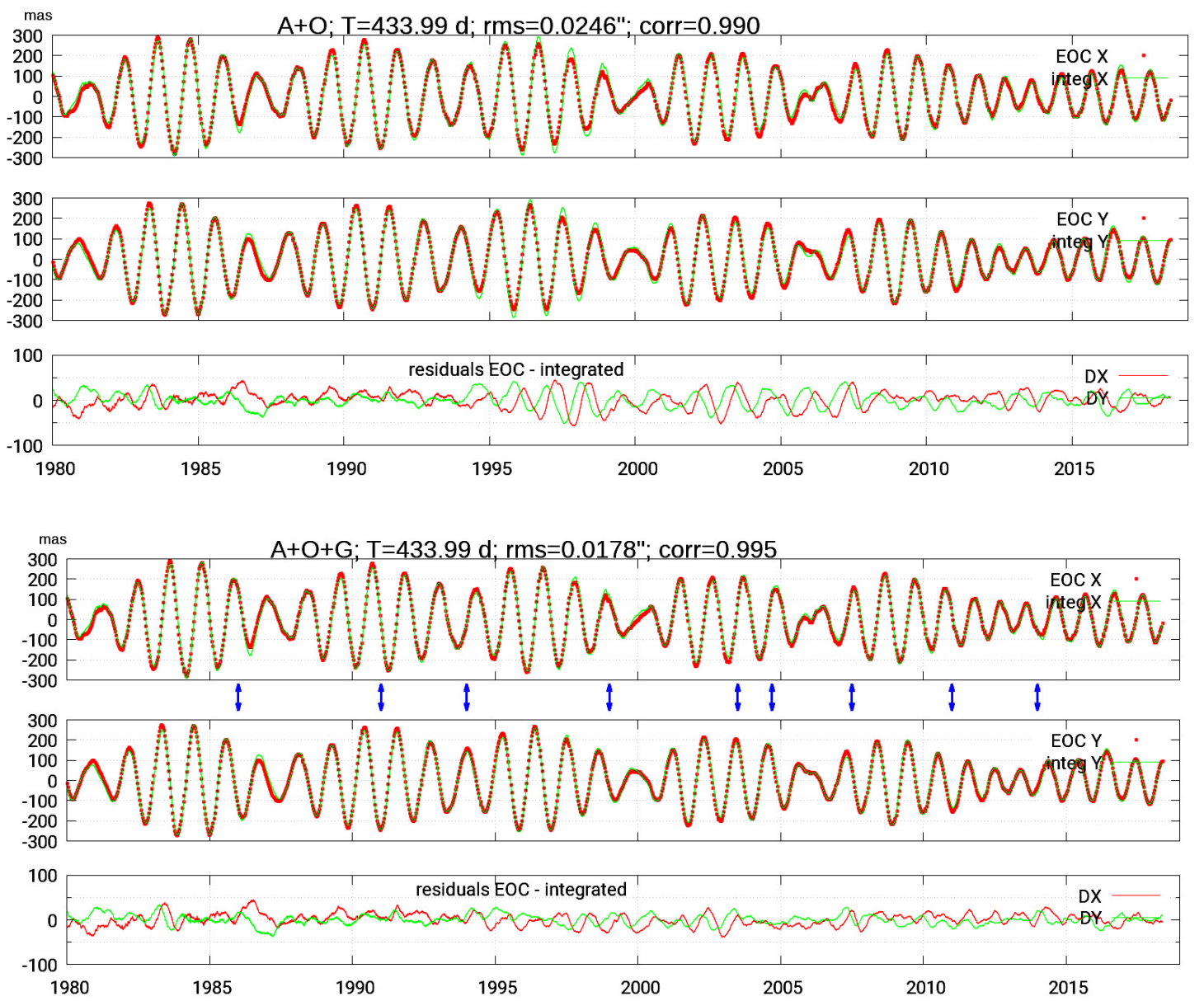

Fig. 1 Comparison of integrated and observed polar motion (dots each 10 days) without GMJ (top) and with GMJ excitation (bottom). The epochs of used geomagnetic jerks are shown as blue arrows. The residuals are shown at bottom graphs.

combinations of period and quality factor of Chandler wobble to get the best rms fit as was described in Vondrák et al. (2017). We show here only the results expressing the improvement caused by the application of GMJ excitation.

It is clearly seen that both series, the observed and integrated one are almost undistinguishable in Figure 1. But, the improvement of the solution with the GMJ excitations is clearly evident in the reduced residual. Therefore we display further only the residuals between integrated and observed (EOC 04) series.

It is seen in Figure 2 that the integration with additional excitations due to hydrospheric and sealevel excitations, although a little bit worse in the whole interval of 1980-2018, is in better agreement with observations in some periods. To show the periods we displayed rms from the residuals in 5-year sliding window at Figure 3 that can be used in testing the quality of these additional excitations in different epochs.

It is seen that solutions with added hydrospheric $(\mathrm{H})$ and the new barystatic sea-level (S) excitations have larger rms in the interval 1984-1995 and in the very last year 2017.
In the earlier years 1980-1990 ECMWF precipitation rates are likely overestimated and less accurate than since 2000. This precipitation errors cause overestimated annual signals in the LSDM water storage and consequently also in hydrospheric EAMF (HAM). As barystatic sea-level EAMF (SLAM) is also determined by the terrestrial water storage, and reflects such overestimated amplitudes. Moreover, HAM and SLAM do not compensate each other on polar motion $\chi_{1}$ and $\chi_{2}$. In contrast, $\chi_{3}$ SLAM totally compensates the annual signal in HAM, thereby suppressing possible errors in HAM coming from the LSDM model.

All four solutions are comparable between years 1995 and 2017. It is also seen that accuracy of all solutions is continuously slightly improving. The power spectrum of the residuals between EOC 04 and $\mathrm{A}+\mathrm{O}+\mathrm{H}+\mathrm{S}+\mathrm{G}$ solutions is displayed at Figure 4. The spectra of the other solutions do not differ substantially. The fortnightly period is very probably a part of EOC 04 rather than the geophysical models. The results of $T$ and $Q$, rms and correlations for different solutions are collected in Table 1. The amplitudes of geomagnetic excitations at GMJ epochs of different solutions are collected in Table 2. The 


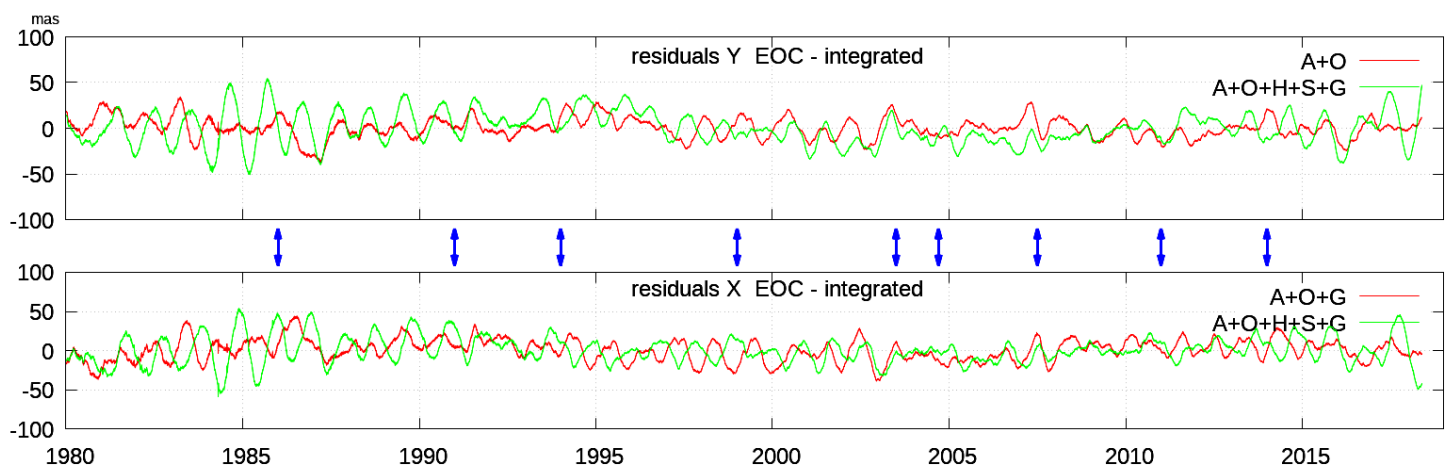

Fig. 2 Residuals of solutions $\mathrm{A}+\mathrm{O}+\mathrm{G}$ and the complete solution $\mathrm{A}+\mathrm{O}+\mathrm{H}+\mathrm{S}+\mathrm{G}$ with respect to the EOC 04.

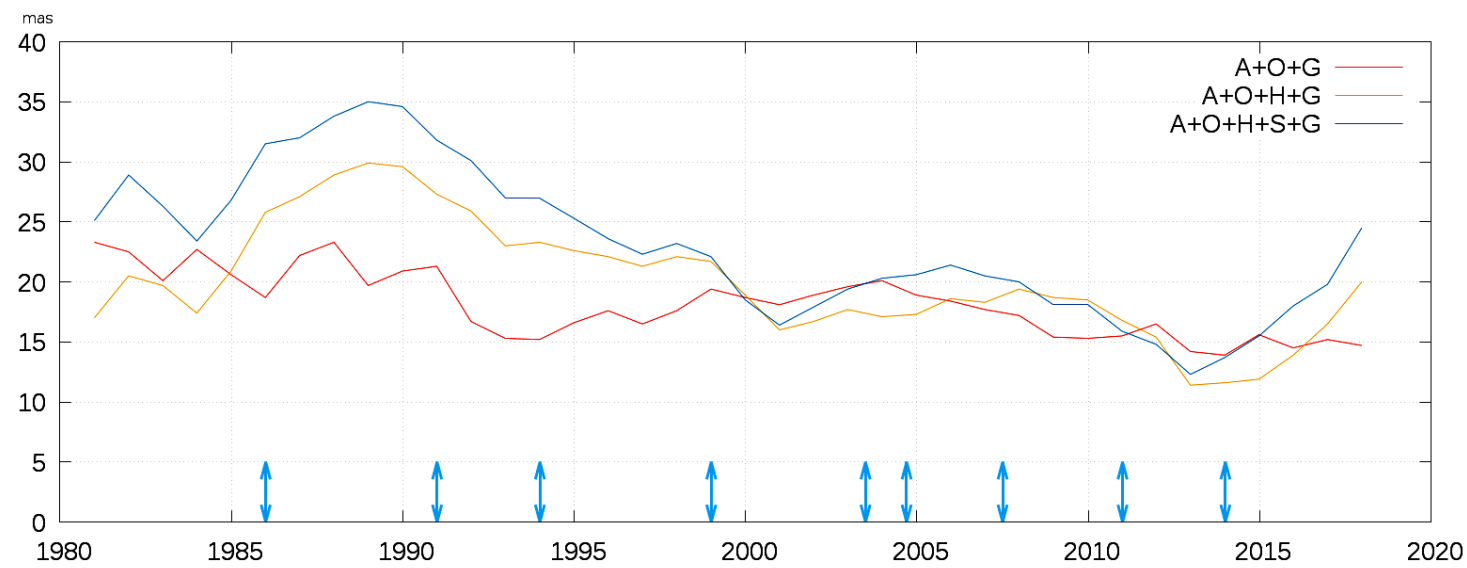

Fig. 3 Comparison of averaged rms errors derived in sliding window 5-year wide and sliding by one year for different solutions.

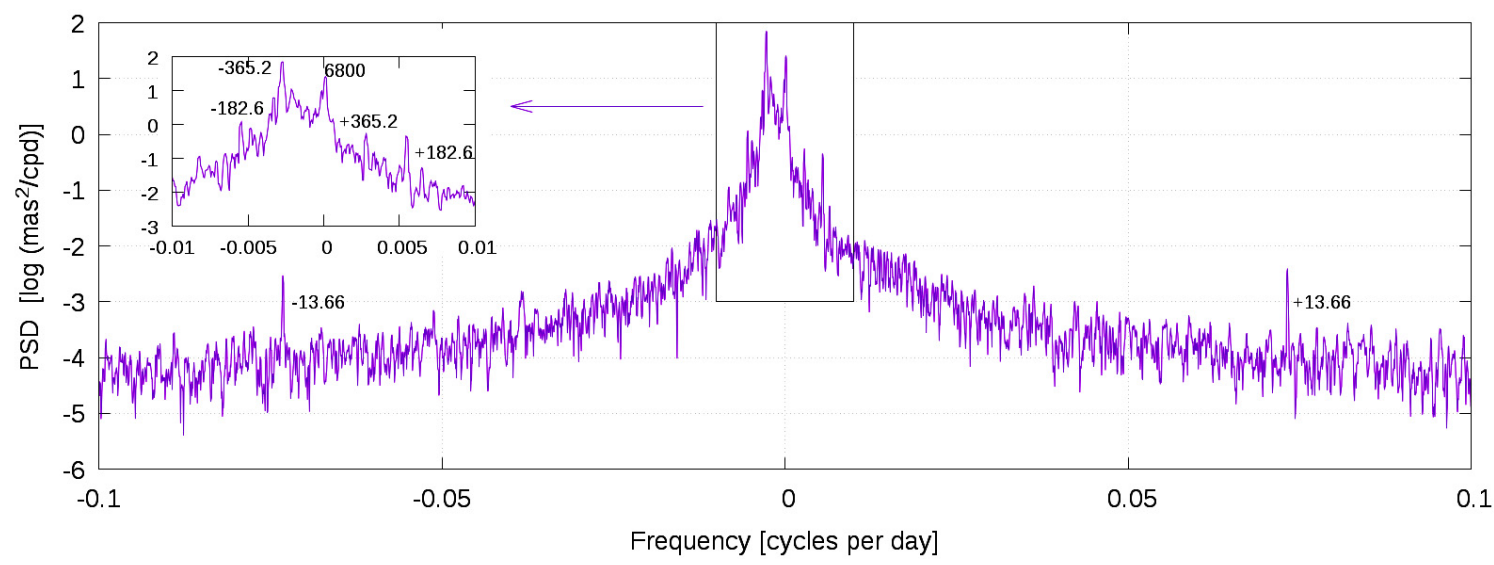

Fig. 4 The power spectral density of residuals between EOC 04 and $\mathrm{A}+\mathrm{O}+\mathrm{H}+\mathrm{S}+\mathrm{G}$ solution. The peaks are marked with periods in days.

values of formal uncertainties of derived parameters $T$ and $Q$ are much smaller than those reported in literature. This can be ascribed partly to the fact that our new results are based on newer and more accurate values of both polar motion and geophysical excitations partly to longer time span used and also due to the new combined method.

The best agreement between the observed and integrated series is obtained in the solution $\mathrm{A}+\mathrm{O}+\mathrm{G}$, with the period of the Chandler wobble $T=433.99$ days, $Q=63.1$, rms $\sigma=17.8$ mas, and correlation with EOC 04 equals to 0.994 . Excitation by hydrosphere is evidently not sufficiently modeled as its inclusion slightly worsens the fit. It also changes the determination of Chandler wobble period, which yields the value by 1 day shorter. Compared to the solution with hydrology only, the addition of barystatic sea-level changes somehow improves the result, but still the best solution is obtained with atmosphere and ocean.

\section{ACKNOWLEDGEMENTS}

This research was financially supported by the project RVO: 67985815 provided by the Czech Academy of Sciences. 
Table 1 List of rms fit (in mas) and correlations with respect to the observed polar motion (EOC 04) and Chandler wobble period $T$ (in days) and quality factor $Q$ of solutions with different combination of excitations. The solution ERA is taken from Vondrák et al. (2017) for comparison (for the interval 1974-2014).

\begin{tabular}{lcccccccc}
\hline & \multicolumn{4}{c}{ without GMJ } & \multicolumn{3}{c}{ with GMJ } \\
\cline { 2 - 8 } Solution & Fit & Corr. & $T$ & $Q$ & Fit & Corr. & $T$ & $Q$ \\
\hline $\mathrm{A}+\mathrm{O}$ & 24.6 & 0.990 & $433.99 \pm 0.03$ & $62.7 \pm 0.5$ & $\mathbf{1 7 . 8}$ & $\mathbf{0 . 9 9 4}$ & $\mathbf{4 3 3 . 9 9} \pm \mathbf{0 . 0 2}$ & $\mathbf{6 3 . 1} \pm \mathbf{0 . 4}$ \\
$\mathrm{A}+\mathrm{O}+\mathrm{H}$ & 29.0 & 0.986 & $432.94 \pm 0.04$ & $58.0 \pm 0.6$ & 21.1 & 0.993 & $433.04 \pm 0.03$ & $58.4 \pm 0.4$ \\
$\mathrm{~A}+\mathrm{O}+\mathrm{H}+\mathrm{S}$ & 31.8 & 0.983 & $432.79 \pm 0.03$ & $58.5 \pm 0.6$ & 24.2 & 0.990 & $432.83 \pm 0.03$ & $58.9 \pm 0.4$ \\
$\mathrm{~A}+\mathrm{O}$ (ERA) & 43.2 & 0.974 & $431.88 \pm 0.04$ & $83 \pm 1$ & 31.1 & 0.986 & $432.86 \pm 0.04$ & $35.0 \pm 0.3$ \\
\hline
\end{tabular}

Table 2 Derived amplitudes of geomagnetic excitations at the GMJ epochs of different solutions in mas.

\begin{tabular}{|c|c|c|c|c|c|c|c|c|c|c|c|c|c|}
\hline \multirow[t]{2}{*}{ Solution } & 1986 & 1991 & 1994 & 1999 & 2003.5 & & 200 & & 200 & & & & 2014 \\
\hline & $\chi_{1} \quad \chi_{2}$ & $\chi_{1} \quad \chi_{2}$ & $\chi_{1} \quad \chi_{2}$ & $\chi_{1} \quad \chi_{2}$ & $\chi_{1} \quad \lambda$ & & $\chi_{1}$ & $\chi_{2}$ & $\chi_{1}$ & $\chi_{2}$ & $\chi_{1}$ & $\chi_{2}$ & $\chi_{1}$ \\
\hline $\mathrm{A}+\mathrm{O}$ & $\begin{array}{ll}-3 & -1 \\
\end{array}$ & 23 & $-27 \quad 4$ & $\begin{array}{ll}-9 & 19\end{array}$ & 191 & 1 & 10. & -21 & 4 & -7 & -1 & 6 & -9 \\
\hline $\mathrm{A}+\mathrm{O}+\mathrm{H}$ & -1 & $\begin{array}{ll}-8 & 10\end{array}$ & $-25-16$ & $\begin{array}{ll}-28 & 21\end{array}$ & 34 & 6 & -7 & 1 & 26 & -15 & -6 & -7 & -14 \\
\hline $\mathrm{A}+\mathrm{O}+\mathrm{H}+\mathrm{S}$ & $\begin{array}{ll}4 & -1\end{array}$ & -9 & $-22-25$ & $-26 \quad 15$ & 42 & 9 & -15 & 10 & 22 & -7 & 1 & -17 & $-7-10$ \\
\hline
\end{tabular}

\section{REFERENCES}

Brown, W. Beggan, C. and Macmillan, S.: 2016, Geomagnetic jerks in the Swarm Era. SP-740 Proceedings of Living Planet Symposium, 9-13 May 2016, Spacebooks Online.

Brzeziński, A.: 1994, Polar motion excitation by variations of the effective angular momentum function: II Extended Model. Manuscr. Geodaet., 19, 157-171.

Chulliat, A. and Maus, S.: 2014, Geomagnetic secular acceleration, jerks, and a localized standing wave at the core surface from 2000 to 2010. J. Geophys. Res. Solid Earth, 119, 1531. DOI: 10.1003/2013B010604

Dill, R.: 2008, Hydrological model LSDM for operational Earth rotation and gravity field variations. Scientific Technical Report No. STR08/09, Potsdam. DOI: 11.2312/GFZ.b103-08095

Dobslaw, H., Dill, R., Grötzsch, A., Brzeziński, A. and Thomas, M.: 2010, Seasonal polar motion excitation from numerical models of atmosphere, ocean, and continental hydrosphere. J. Geophys. Res., 115, B10406. DOI: 10.1029/2009JB007127

Dobslaw, H. and Dill, R.: 2018, Predicting Earth orientation changes from global forecasts of atmospherehydrosphere dynamics. Adv. Space Res., 61, 4, 10471054. DOI: $10.1016 /$ j.asr.2017.11.044

Dobslaw, H. and Dill, R.: 2019, Product Description Document, EAMF from ESM at GFZ in Potsdam. ftp://esmdata.gfz-potsdam.de/EAM.

Gross, R.S.: 2005, The observed period and Q of the Chandler wobble. In. Plag, H.-P., Chao, B.F., Gross, R.S. and van Dam, T. (Eds.), Forcing of polar motion in the Chandler frequency band. Cahiers du CEGS, Luxembourg, 24, 31-37.

Gross, R.S.: 2009, Earth rotation variations - long period. In: Schubert, G., Herring, T. (Eds.), Treatise on Geophysics, vol. 3. Geodesy, Elsevier, 239-294.

Jungclaus, J.H., Fischer, N., Haak, H., Lochmann, K., Marotzke, J., Matei, D., Mikolajewics, U., Notz, D. and von Storch, J. S.: 2013, Characteristics of the ocean simulations in the Max Planck Institute Ocean Model (MPIOM) the ocean component of the MPIEarth system model, J. Adv. Model. Earth Sys., 5, 422-446. DOI: $10.1002 /$ jame. 20023

Koot, L. and de Viron, O.: 2011, Atmospheric contribution to nutation and implications for the estimate of deep Earth's properties from nutation observations.
Geophys. J. Int., 185, 3, 1255-1265.

DOI: $10.1111 /$ j.1365-246X.2011.05026.x

Malkin, Z.: 2013, Free core nutation and geomagnetic jerks. J. Geodyn., 72, 53-58.

DOI: $10.1016 /$ j.jog.2013.06.001

Mandea, M., Holme, R., Pais, A., Pinheiro, K., Jackson, A. and Verbanac, G.: 2010, Geomagnetic jerks. Rapid core field variations and core dynamics. Space Sci. Rev., 155, 147-175.

DOI: 10.1007/s11214-010-9663-x

Olsen, N. and Mandea, M.: 2008, Rapidly changing flows in the Earth's core. Nat. Geosci., 1, 6, 390-394. DOI: $10.1038 /$ ngeo203

Tamisiea, M.E., Hill, E.M., Ponte, R.M., Davis, J. L., Velicogna, I., and Vinogradova, N.T.: 2010, Impact of self-attraction and loading on the annual cycle in sea level. J. Geophys. Res. Oceans, 115(C7), 1-15. DOI: $10.1029 / 2009 J C 005687$

Thomas, M.: 2002, Ocean induced variations of Earth's rotation - Results from a simultaneous model of global circulation and tides. Ph.D dissertation, University of Hamburg, Germany, 129 pp.

Vondrák, J.: 1977, Problem of smoothing observational data II. Bull. Astron. Inst. Czechosl., 28, 84-89.

Vondrák, J. and Ron, C.: 2015, Earth orientation and its excitations by atmosphere, oceans, and geomagnetic jerks. Serb. Astron. J., 191, 59-66. DOI: $10.2298 / \mathrm{SAJ} 1591059 \mathrm{~V}$

Vondrák, J. and Ron, C.: 2016, Geophysical fluids from different data sources, geomagnetic jerks, and their impact on Earth's orientation. Acta Geodyn. Geomater., 13, 3, 241-247. DOI: 10.13168/AGG.2016.0005

Vondrák, J. and Ron, C.: 2017, New method for determining free core nutation parameters, considering geophysical effects. Astron. Astrophys., 604, A56. DOI: $10.1051 / 0004-6361 / 201730635$

Vondrák, J. and Ron, C.: 2019, New GFZ effective angular momentum excitation functions and their impact on nutation. Acta Geodyn. Geomater., 16, 2, 151-155. DOI: 10.13168/AGG.2019.0012

Vondrák, J., Ron, C. and Chapanov, Ya.: 2017, New determination of period and quality factor of Chandler wobble, considering geophysical excitations. Adv. Space Res. 59, 1395-1407. DOI: 10.1016/j.asr.2016.12.001 